\title{
On Optimality, Endogenous Discounting and Wealth Accumulation
}

I. Schumacher

Discussion Paper 2006-58

\section{Département des Sciences Économiques de l'Université catholique de Louvain}


CORE DISCUSSION PAPER

$2006 / 103$

\title{
ON OPTIMALITY, ENDOGENOUS DISCOUNTING AND WEALTH ACCUMULATION
}

\author{
Ingmar SCHUMACHER ${ }^{1}$
}

$11 / 2006$

\begin{abstract}
We endogenize the discount rate via a broad measure of wealth and provide empirical evidence that wealth affects the discount rate negatively.

We demonstrate that the Pontryagin conditions require positive felicity for intuitive results, whereas the concavity of the Hamiltonian requires negative felicity for optimality. This dilemma also holds for the endogenizations of Obstfeld (1990) and followers.

We solve the model with positive felicity and resolve when optimality is possible. We discuss the impact on technological change, savings and convergence which are more in line with empirics. Finally, we discuss time consistency of a planner who cannot predict his preferences.
\end{abstract}

JEL Classification: D90, C61, O41.

Keywords: endogenous time preference, stability, optimal growth, recursive utility.

\footnotetext{
${ }^{1}$ Center for Operations Research and Econometrics (CORE), Université catholique de Louvain, Belgium. E-mail: schumacher@core.ucl.ac.be.

The author kindly acknowledges financial support from the Chaire Lhoist Berghmans in Environmental Economics. I am indebted to Jakub Growiec and Raouf Bouccekine for various comments. I also thank Agustin Perez and Claude d'Aspremont for discussions. Obviously, if you find an error then let me know because it belongs to me.
} 


\section{Introduction}

Impatience is known to be one of the key parameters affecting our consumption decisions over time. It is now widely accepted that too little valuation of future streams of utility can result in choosing a path of consumption which might have consumption decreasing over time and thus sacrifice future generations for the sake of high current consumption. Conversely, if current generations value the utility of future generations by too much, then this requires excessive sacrifices from the current generation. At the heart of this debate is, of course, the commonly raised question of what determines the actual level of the discount rate. Classical growth models approach this question by suggesting that the discount rate is a mixture consisting of fashion, foresight, self-control, life-expectancy, habit and a concern for others (Fisher 1930). However, they treat the discount rate in a somewhat simplistic manner as they do not allow for changes in it.

The appropriateness of the use of exogenous discount rates in the standard macroeconomic growth models has recently been questioned by numerous empirical studies (Frederick et al. 2002, Lawrence 1991, Samwick 1998, Portney and Weyant 1999, etc.). As far as empirical evidence is concerned, Lawrence (1991) finds that the discount rate of poor households is up to $5 \%$ higher than that of rich households. Similary, Samwick (1998) observes that the discount rate declines with the income level, and finally Frederick et al. (2002) conclude in another empirically-founded review that increased wealth can influence the discount rates. The empirical evidence therefore clearly concludes that wealth is decisive for the weight we give to the future in current decisions.

Naturally, the past few years have seen a recurring interest in theoretical models with intertemporally dependent preferences which attempt to investigate the key consequences of various sources of endogeneity in the discount rate (Becker and Mulligan 1997, Epstein and Hynes 1983, Obstfeld 1990, Das 2003, Pittel 2002, Ayong Le Kama and Schubert 2003). These models nearly exclusively deal with consumption, a flow variable, as the source of endogenous discounting. They also, nearly exclusively, utilize a discount rate which is an increasing function of consumption. Our intention is to depart from that literature and to choose a stock variable which affects the discount rate. This is interesting for several reasons.

Firstly, it is more in line with the empirical evidence forwarded by the literature on endogenous discounting. Specifically, we argue that wealth affects the discount rate by proposing that this is a shortcut for suggesting that wealthier countries have better health standards (Case et al. 2002, Pritchett 1996) and better insurance markets (Caroll 1997, Banerjee and Newman 1991), both of which generally affects 
the agent's discount rate negatively (Frederick et al. 2002). We thus propose that the stock of wealth is assumed to affect the level of the discount rate negatively. This leads to a departure from the time-additive framework of Koopmans (1960) to the recursive framework poineered by Uzawa (1968).

Secondly, utilizing a stock variable has interesting theoretical repercussions as the steady state level is affected to a different extend than in the previous models. Furthermore, the theoretical analysis here will help to solve and better understand future models which are built in such a way that the discount rate is endogenized via a state variable, as the effects of this type of endogenization will be generic for all stock-type endogenizations.

Another relevant addition of this article to the literature is the discussion of the necessary and sufficient conditions for optimality in the case of a decreasing endogenous discount rate. To be precise, we will show that there exists a generic incompatibility in continuous time models of endogenous discounting between the requirements imposed by the sufficiency conditions and the endogenization of the discount rate. Specifically, we demonstrate that, on the one hand the concavity conditions of the Hamiltonian require negative utility, a result equivalent to Obstfeld (1990). To compare to Obstfeld (1990) and others we use the Mangasarian conditions to assess concavity of the Hamiltonian, although the Arrow and Kurz conditions give the same requirement. On the other hand, and this seems to have been unnoticed in the previous literature, the Pontryagin necessary conditions require positive utility for intuitively plausible results. We show that disregarding the requirement of negative utility imposed by the Mangasarian conditions can be a way out of this dilemma. As we will argue this leads to intuitively plausible conclusions. Most importantly, we will use two methods to demonstrate why an optimal program exists. The first argument is based on the property of saddle-path stability coupled with the Michel (1982) transversality condition and the second one utilizes Bellman's principle of optimality. We then use a simulation in order to further support our argument that positive felicity indeed results in an optimum.

In addition, we draw some policy implications from changes in technology as well as the effect of the endogenous discount rate on savings and convergence speed. The final remark will be on time consistency.

The article is organized as follows. The next section introduces some empirical backgrounds. The following section 3 introduces the model. We then discuss about necessary and sufficient conditions in sections 4 and 5. Section 6 reduces the system and in section 7 we introduce the conflict between Mangasarian requirements and intuition. In the next section we get rid of the Mangasarian condition and show how this brings the model in line with intution, followed by a discussion of optimality and an 
analysis of the model. Finally, the conclusion.

\section{Empirical background}

Is this feedback, from wealth to patience, a viable one? In this section we review some empirical evidence supporting this hypothesis.

As suggested above, our treatment of wealth here will be based upon a broad view of wealth by taking a combination of physical capital and including human capital (see e.g. Barro and Sala-i-Martin 1995). This will allow to place the model in a wider context. ${ }^{1}$

The hypothesis that there exist some factors which shape our preferences can already be traced back to Böhm-Bawerk (1889) and Fisher (1930). Böhm-Bawerk believed that any analysis of intertemporal choice ought to include the "effective desire of accumulation". He therefore proposed several objective as well as subjective factors which he believed to be the underlying psychological determinants of time preference. The two main objective factors were the projected future wealth as well as risk. This was later supported by Fisher, who suggested that "poverty bears down heavily on all portions of a man's expected life. But it increases the want for immediate income even more than it increases the want for future income." (1930, pp.732) As our analysis will be based upon an infinitely-lived agent approach, we shall concentrate on the two factors noted above, wealth and risk. ${ }^{2}$ We can therefore introduce the following determinants of the discount rate $(\rho)$, consisting of physical wealth $(\mathrm{PW})$, human wealth $(\mathrm{HW})$, mortality (M) and other risks (OR):

$$
\rho=f(\underbrace{\mathrm{PW}}_{(-)}, \underbrace{\mathrm{HW}}_{(-)}, \underbrace{\mathrm{M}}_{(+)}, \underbrace{\mathrm{OR}}_{(+)})
$$

The effect of the variables on the discount rate is clear even in the early writings - wealth decreases the discount rate and risk increases it. In the subsequent paragraphs we shall be more specific and investigate the relationship between the variables determining the discount rate itself.

We shall firstly take a look at the effect of physical and human wealth on the discount rate and the variables in question. Fielding and Torres (2005) estimate the strength of the relationship between several factors, including wealth, health and education for case of 41 developing countries. Their measure of

\footnotetext{
${ }^{1}$ For an endogenous discounting model which separates human capital from physical capital see Fall and Schumacher (2006).

${ }^{2}$ Frederick et al. (2002) have an excellent survey with more reference to the subjective factors.
} 
wealth is of particular usefulness here, as it is based upon a broader measure of wealth than standard empirical analysis that only refers to income. They build an index of wealth which includes variables like presence of an electricity supply, possession of radio, TV or car, flush toilet, etc. Then they estimate that improvements in physical wealth and improvements in human wealth lead to lower mortality rates. Important is that their results are robust even across countries, pointing at a rather uniform effect of the variables in question.

This finding has been confirmed over and over again. Grossman (2003) and Grossman and Kaestner (1997) review the literature on the relationship between human wealth and health. Their main conclusion suggests that the variable which has the highest correlation with health is human wealth. To give some magnitude to this effect, Elias (2003) has related the death rates to the educational attainments for the USA for white males. From the 45 to 64 years old who have less than 12 years of schooling, the death per 100,000 inhabitants were 1304, whereas the deaths for those who have more than 13 years of schooling was less than half, 510 people. These findings also seem robust over time. Similary, Kitagwa and Hauser (1973) utilizing data from the 1960 Census Records for the USA conclude that, even after controlling for income, human wealth has a significant negative relationship with mortality rates. Based on different data, these results are confirmed by Feldman et al. (1989), Pappas et al. (1993), Preston and Elo (1995), Richard and Barry (1998), and many more.

Therefore, income alone cannot be the reason for a lower mortality rate. This therefore excludes the possibility that a person with higher income can simply pay his way out of illness. We therefore have to search for underlying variables which shape the behaviour of people in such a way that they act so as not to incur the illness in the first place.

Clearly an important feedback comes through time preference itself. If I am more patient then I put more weight on future outcomes today. Therefore, assuming I do not care about my future (ie. a high discount rate), the high likelihood of developing cancer in old age will not figure highly in my decision today to chain smoke. On the contrary, a high preference for the future will make me look cancer directly in the eye and will most likely stop me from smoking. It has now been widely proposed that human wealth has this large influence on time preference. There exists a strong literature suggesting that higher human wealth reduces smoking (e.g. de Walque 2004, Sander 1995, Kenkel 1991) and obesity (Nayga 2001), thus has a negative impact on mortality. We can therefore write:

$$
\rho=f(\underbrace{\mathrm{PW}}_{(-)}, \underbrace{\mathrm{HW}}_{(-)}, M(\mathrm{HW}, \mathrm{PW}), \underbrace{\mathrm{OR}}_{(+)})
$$


Wealth as a possible source of endogenous discounting has also been proposed by Becker and Mulligan (1997) who conclude that financial asset inequality grows as cohorts age, a sign that financial assets affect preferences itself. Similarly, Deaton and Paxson (1994) show that human capital inequality grows at cohorts age, again a sign that human capital affects preferences.

Other arguments for a broad wealth effect on discounting can be forwarded on intuitive grounds. For example, if John expects being rich when old, then John will surely look forward to this situation. However, if he expects to be poor, he will prefer not to think about the future at all. Put differently, if one expects not to get anything out of life, then one will prefer not to think about the future at all.

Similar reason can be forwarded based on an argument by Parfit (1984). Parfit suggests that "we care less about our future because we know that less of what we are now... will survive into the further future." Discounting thus reflects the expected change in personal identity. If I compare myself with my possible future self when taking decisions, then I might be inclined to ask: If my future self is rich, then am I going to look forward to being this future self? If I am thus looking forward to a future self which is wealthier, then I will be more inclined to associate myself with this future self and thus put more weight on this future self in my current decisions, than if this future self is poor.

Our analysis therefore allows to conclude the following determinants of the discount rate, where we combine $P W$ and $H W$ into $k$ :

$$
\rho=f(\underbrace{\mathrm{k}}_{(-)}, M(\underbrace{\mathrm{k}}_{(-)}), \underbrace{\mathrm{OR}}_{(+)}) \quad \Longrightarrow \quad \rho=\rho(k)
$$

In the subsequent analysis we shall simply take the other risks and factors as exogenously given and allow them to determine an exogenous level of the discount rate below which the discount rate cannot fall, $\hat{\rho}$. Therefore, the determinant of the discount rate which we focus on is a combination of physical as well as human wealth, which gives a broad measure of wealth as in Mankiw (1995) or Barro and Sala-i-Martin (1995).

\section{The Model}

The model is based on an infinitely-lived agent approach where the agent obtains utility from consumption. In addition, his wealth affects the discount rate negatively. Then, wealth can be accumulated by investing but is reduced by consumption and constant depreciation. The infinitely-lived agent then 
attempts to solve the following problem.

$$
\begin{aligned}
& \max _{\{c(t)\}} U(c(t), k(t))=\int_{t=0}^{\infty} u(c(t)) e^{-\theta(t)} d t \\
& \begin{cases}\dot{k}(t)=f(k(t))-c(t)-\delta k(t), & \forall t \\
\dot{\theta}(t)=\rho(k(t)), & \forall t \\
k(t) \geq 0, c(t) \geq 0, & \forall t, \\
\text { with } k(0) \text { given. }\end{cases}
\end{aligned}
$$

Assumption 1 We impose that the production function $f: \mathbb{R}_{+} \rightarrow \mathbb{R}_{+}$follows standard assumptions of concavity, such that $f(0)=0, f^{\prime}(k)>0, f^{\prime \prime}(k)<0$. We also impose the standard Inada conditions, given by $\lim _{k \rightarrow 0} f^{\prime}(k)=\infty$, and $\lim _{k \rightarrow \infty} f^{\prime}(k)=0$. Finally, we assume that $f^{-1}$ exists and is unique.

Assumption 2 The utility function $u: \mathbb{R}_{+} \rightarrow \mathbb{R}$ is at least twice continuously differentiable and has the standard properties of $u^{\prime}(c)>0, u^{\prime \prime}(c)<0, \forall c$. We assume $u^{\prime}(0)=\infty$.

The assumption $u^{\prime}(0)=\infty$ allows to concentrate on interior solutions only. It corresponds to the assumption that at least a minimum amount of consumption is required for the continuation of the generations. We shall also sometimes resort to the constant relative risk aversion (CRRA) utility function, which has the functional form of $u(c)=\frac{c^{1-\sigma}}{1-\sigma}$, with $\sigma \geq 0$.

Assumption 3 We assume that the at least twice differentiable discount rate $\rho(k): \mathbb{R}_{+} \rightarrow \mathbb{R}_{++}$, has the properties $\lim _{k \rightarrow \infty} \rho(k)=\hat{\rho}>0, \rho^{\prime}(k)<0, \rho^{\prime \prime}(k)>0$, and $\rho^{\prime}(k)<\infty, \forall k$.

The assumption on the production and utility function are standard and do not require any justification. The assumption for the discount rate has been motivated in the previous section. Defining the optimization problem by introducing the discount factor as another constraint allows the Hamiltonian to be independent of time which greatly simplifies the analysis. ${ }^{3}$

\section{Necessary Conditions}

The Hamilton of the above system writes

$$
\mathcal{H}=u(c(t)) e^{-\theta(t)}+\lambda(t)[f(k(t))-c(t)-\delta k(t)]-\mu(t) \rho(k(t)) .
$$

\footnotetext{
${ }^{3}$ Throughout the article we use $x^{\prime}(y)=\partial x / \partial y, \dot{x}=\partial x / \partial t$.
} 
The Pontryagin necessary conditions for optimality are

$$
\begin{aligned}
u^{\prime}(c(t)) e^{-\theta(t)} & =\lambda(t), \\
\lambda(t)\left[f^{\prime}(k(t))-\delta\right]-\mu(t) \rho^{\prime}(k(t)) & =-\dot{\lambda}(t), \\
-u(c(t)) e^{-\theta(t)} & =\dot{\mu}(t), \\
\lim _{t \rightarrow \infty} \mathcal{H}(t) & =0,
\end{aligned}
$$

where equation (9) gives the transversality condition of the system. ${ }^{4}$ As $\mu(t)$ represents the implicit value of relaxing the constraint (5) by one unit, we expect that $\mu(t)$ should give us the prospective, discounted value of changes in the discount rate. We are able to confirm this as after integrating equation (8) we obtain

$$
\mu(t)=\int_{t}^{\infty} u(c(s)) e^{-\theta(s)} d s
$$

This result is of course only valid if the utility functional converges to zero when time goes to infinity, which will be the case when utility is finite and the discount rate is positive. Hence we know that $\mu(t)<(>) 0$ if $u(c(t))<(>) 0$, which is important for the second-order sufficiency conditions.

\section{Sufficiency Conditions}

To assess the concavity of the Hamiltonian we make use of the Mangasarian conditions. However, even the weaker Arrow and Kurz conditions have the same requirement on utility. We state the result in the next proposition.

Proposition 1 Given the optimization problem (4) a necessary condition for the Mangasarian sufficient conditions to be satisfied is given by $u(c(t)) \leq 0$.

Proof 1 The second order sufficiency conditions are derived from the following matrix

$$
M=\left[\begin{array}{ccc}
u^{\prime \prime}(c(t)) e^{-\theta(t)} & 0 & -u^{\prime}(c(t)) e^{-\theta(t)} \\
0 & \lambda(t) f^{\prime \prime}(k(t))-\mu(t) \rho^{\prime \prime}(k(t)) & 0 \\
-u^{\prime}(c(t)) e^{-\theta(t)} & 0 & u(c(t)) e^{-\theta(t)}
\end{array}\right]
$$

\footnotetext{
${ }^{4}$ For this kind of transversality condition, see Michel (1982).
} 
The requirement for negative definiteness is that the principal minors alternate in sign. The principal minors are given by

$$
\begin{aligned}
& \left|M_{1}\right|=u^{\prime \prime}(c(t)) e^{-\theta(t)} \\
& \left|M_{2}\right|=u^{\prime \prime}(c(t)) e^{-\theta(t)}\left[\lambda(t) f^{\prime \prime}(k(t))-\mu(t) \rho^{\prime \prime}(k(t))\right] \\
& \left|M_{3}\right|=\left[u^{\prime \prime}(c(t)) u(c(t))-u^{\prime}(c(t))^{2}\right] e^{-2 \theta(t)}\left[\lambda(t) f^{\prime \prime}(k(t))-\mu(t) \rho^{\prime \prime}(k(t))\right] .
\end{aligned}
$$

Given our regularity conditions on the utility function we know that $\left|M_{1}\right|<0$. Hence, $\left|M_{2}\right|$ must be positive, which only holds if $\lambda(t) f^{\prime \prime}(k(t))-\mu(t) \rho^{\prime \prime}(k(t))<0$. Given $\lambda>0$ and $f^{\prime \prime}(k(t))<$ 0 , a necessary condition is that $\mu(t) \rho^{\prime \prime}(k(t))>\lambda(t) f^{\prime \prime}(k(t))$. Given that the principal minors must alternate in sign we must have that $\left|M_{3}\right|<0$. Given the requirement on $\left|M_{2}\right|$, this necessarily imposes $u^{\prime \prime}(c(t)) u(c(t))-u^{\prime}(c(t))^{2}>0$. Hence, a necessary condition is that $u(c(t)) \leq 0$

In the case of a CRRA utility function, this condition is also sufficient. Given that we must impose $u(c(t)) \leq 0$, this implies that $\mu(t)<0, \forall t$. Hence, For $\rho^{\prime \prime}(k)>0$ the combination of $u(c(t)) \leq 0$ and $\mu(t) \rho^{\prime \prime}(k(t))>\lambda(t) f^{\prime \prime}(k(t))$ are sufficient conditions for optimality.

\section{Solving the Model}

Transforming the Pontryagin necessary conditions from (6) till (8) and disregaring time subscripts for convenience, we arrive at the following system of dynamical equations:

$$
\begin{aligned}
\dot{c} & =-\frac{u^{\prime}(c)}{u^{\prime \prime}(c)}\left[f^{\prime}(k)-\delta-\rho(k)-\frac{\mu}{u^{\prime}(c) e^{-\theta}} \rho^{\prime}(k)\right], \\
\dot{k} & =f(k)-c-\delta k, \\
\dot{\mu} & =-u(c) e^{-\theta} .
\end{aligned}
$$

It is possible to reduce this system to a system in two dynamical equations, only. This we do as follows. The first observation is that the Hamiltonian of the above system is autonomous. We can therefore show the following. As we have that $\mathcal{H}=u(c) e^{-\theta}+\lambda \dot{k}-\mu \dot{\theta}$, then we know that necessary conditions for optimality are $\frac{\partial \mathcal{H}}{\partial c}=0, \frac{\partial \mathcal{H}}{\partial k}=-\dot{\lambda}, \frac{\partial \mathcal{H}}{\partial \theta}=\dot{\mu}$, as well as $\frac{\partial \mathcal{H}}{\partial \lambda}=\dot{k}$ and finally $\frac{\partial \mathcal{H}}{\partial \mu}=\dot{\theta}$. Taking partial differentials of the Hamiltonian with respect to time we obtain $\frac{d \mathcal{H}}{d t}=\frac{\partial \mathcal{H}}{\partial t}+\frac{\partial \mathcal{H}}{\partial c} \dot{c}+\frac{\partial \mathcal{H}}{\partial k} \dot{k}+$ $\frac{\partial \mathcal{H}}{\partial \theta} \dot{\theta}+\frac{\partial \mathcal{H}}{\partial \lambda} \dot{\lambda}-\frac{\partial \mathcal{H}}{\partial \mu} \dot{\mu}$. Given the conditions for optimality we can cancel out and are left with $\frac{d \mathcal{H}}{d k}=\frac{\partial \mathcal{H}}{\partial t}$. As the Hamiltonian is autonomous, we also have that $\frac{\partial \mathcal{H}}{\partial t}=0$. Given the transversality condition $\lim _{t \rightarrow \infty} \mathcal{H}(t)=0$, this gives us that the optimized $\mathcal{H}^{*}(t)=0, \forall t$. 
Hence we can transform the Hamiltonian to

$$
\mu=\frac{u(c) e^{-\theta}+u^{\prime}(c) e^{-\theta} \dot{k}}{\rho(k)},
$$

which we then can substitute in the system of equations (14) - (15) to get the following system

$$
\begin{aligned}
\dot{c} & =-\frac{u^{\prime}(c)}{u^{\prime \prime}(c)}\left[f^{\prime}(k)-\delta-\rho(k)-\frac{\rho^{\prime}(k)}{\rho(k)}\left(\frac{u(c)}{u^{\prime}(c)}+\dot{k}\right)\right], \\
\dot{k} & =f(k)-c-\delta k .
\end{aligned}
$$

This reduced-form system captures the complete dynamics of the system. From this we go on to discuss the effect of imposing the Mangasarian sufficiency conditions, as given by $u(c)<0$.

\section{The problem of negatice felicity}

For $\rho^{\prime}(k)<0$ it is possible to show that a steady state always exists, although it might be a unique or a multiple steady state. Then, it is also possible to demonstrate that the system is saddle-path stable if a certain curvature condition on the production function is satisfied. Without providing further proof of the required conditions for existence of steady states as well as the dynamics, we are now going to show the problem of using negative utility with endogenous discounting models. First, we take the steady state version of equations (18), given by

$$
f^{\prime}(k)-\delta=\rho(k)+\frac{\rho^{\prime}(k)}{\rho(k)} \frac{u(c)}{u^{\prime}(c)} .
$$

In the original Ramsey case, we have $\rho^{\prime}(k)=0, \forall k$. Therefore, the smaller is $\rho$, the closer is the optimal capital stock to the Golden Rule level. ${ }^{5}$ Hence, impatience is something "bad" in a sense, which keeps one from attaining the highest possible level of consumption. ${ }^{6}$ So now, if we take the case of $\rho^{\prime}(k)<0$, then we would expect that a policy maker would try to increase optimal capital stock, as this brings him closer to the Golden Rule. This means that increases in capital both decrease the level of discounting and increase the level of consumption over time. However, given we impose the Mangasarian sufficiency conditions, namely $u(c)<0$, we directly see that the last term on the right-hand side is positive. Hence, negative utility and a decreasing discount rate, which should be intuitively favourable towards the golden

\footnotetext{
${ }^{5}$ The Golden Rule level is the maximum sustainable consumption level, which in this case is where $f^{\prime}(k)=\delta$.

${ }^{6}$ For early references on critical approaches towards impatience c.f. Marshall (1890), Pigou (1920) or Ramsey (1928).
} 
rule, make us choose a steady state further away from the golden rule than the exogenous discounting case. This is certainly in conflict with intuition. On the converse (and for the purpose of better illustration only), an increasing discount rate would take us closer to the golden rule and can even make us choose a steady state at the golden rule (if $\rho(\bar{k})=\frac{\rho^{\prime}(\bar{k}) u(\bar{c})}{\rho(k) u^{\prime}(\bar{c})}$ ) or make us choose a higher level of capital stock than the golden rule level. Again, this seems to be at contradiction with the standard Ramsey exercise, where lower discount rates are necessary to take us closer to the golden rule, and not increasing discount rates. Especially in the case of a discount rate decreasing with the level of capital, one would expect that there is incentive to accumulate more capital than in the exogenous discounting case. What goes wrong?

The easy answer first. As can be seen, the last term on the right-hand side is positive for $\rho^{\prime}(k)<0$ if $u(c)<0$. So, negative utility seems be the source of the problem. ${ }^{7}$

To provide more foundation to this result we transform equation (14), by substituting for $\mu(t)$ its optimal value, which is the discounted prospective value of utility on the optimal path. We therefore obtain

$$
\dot{c}(t)=-\frac{u^{\prime}(c)}{u^{\prime \prime}(c)}\left[f^{\prime}(k(t))-\delta-\rho(k(t))-\frac{\rho^{\prime}(k(t)) \int_{t}^{\infty} u(c(s)) e^{-\theta(s)} d s}{u^{\prime}(c(t)) e^{-\theta(t)}}\right]
$$

We are going to analyze the last part of this equation, which helps us in understanding the paradox raised in the previous paragraphs:

$$
\frac{-\rho^{\prime}(k(s)) e^{-\int_{0}^{t} \rho(k(s)) d s} \int_{t}^{\infty} u(c(s)) e^{-\int_{t}^{s} \rho(k(\tau)) d \tau} d s}{u^{\prime}(c(t)) e^{-\int_{0}^{t} \rho(k(s)) d s}} .
$$

The term above is the ratio of two Volterra derivatives. The numerator of this ratio is the Volterra derivative of the utility functional with respect to capital. The denominator is the Volterra derivate with respect to consumption, which is always positive, implying that higher consumption has a positive marginal effect on the utility functional. Given our requirements imposed by the Mangasarian second-order conditions of negative utility the numerator is negative, suggesting that higher capital reduces overall utility. Thus, in the case of endogenous discounting with negative utility, discounting becomes something "good" in the sense that higher discounting actually increases utility. This, of course, is the result of having the level of utility itself in the Volterra derivative. We therefore do not only have to look at marginal changes, but also at the sign of utility. Conclusively, we lose invariance with respect to affine transformations. Specifically, utility functions give rise to opposite conclusions if utility is either negative or positive. However, as utility must be negative for sufficient conditions to hold, we are left with a

\footnotetext{
${ }^{7}$ I am most grateful to Jakub Growiec for providing the key hint for this.
} 
dilemma. Negative utility is required by the Mangasarian conditions, whereas it leads to counterintuitive implications from the necessary condition. Apparently we have to dispose of one of the assumptions.

Here we would like to point at the way the current literature deals with this situation. Firstly, Obstfeld (1990) notices that sufficiency conditions require negative utility and then analyzes the model with a discount rate increasing in consumption. Clearly, his analysis is subject to the same criticism, as an increasing discount rate with a negative utility gives incentive to the policy maker to increase consumption as higher discounting increases prospective utility if utility is negative. The same requirements are imposed by Epstein (1987), Shi and Epstein (1993), to name just a few of the articles.

Ayong le Kama and Schubert (2005) impose a negative utility for sufficiency conditions and assume that the discount rate increases in the level of environmental quality. Similarly, Pittel (2002) assumes that the discount rate is a function of pollution and imposes a negative utility. The same reasoning applies as before, as the policy maker has an incentive to increase environmental quality simply because it leads to a higher discount rate and thus a level of utility closer to zero, which is the upper bound of the utility function which they assume.

Das (2003) uses a discount rate decreasing in the level of consumption and assumes positive utility throughout. She does not talk about Mangasarian conditions, knowing that they will not hold given her assumption on utility. ${ }^{8}$

Overall, it seems that assuming negative utility is required for sufficiency by the second-order conditions, but leads to counterintuitive results. This is one unfortunate moral of this article. However, it by no means is the end of the story. It seems that the Mangasarian condition is indeed only sufficient for optimality, but not necessary.

\section{The case of $u(c) \geq 0$}

We shall now analyze what happens when we disregard the requirement of the second-order conditions. We therefore assume that utility is non-negative on its whole domain, such that $u: \mathbb{R}_{+} \rightarrow \mathbb{R}_{+} \cdot{ }^{9}$ In this case we only know - for now - that the first-order conditions are necessary, not sufficient. We will obtain

\footnotetext{
${ }^{8}$ However, to be fair, her solution is still optimal. Nevertheless, she does not seem to be aware of the problem imposed by negative utility.

${ }^{9}$ This implies that the utility function is variant to affine transformations, which we therefore must rule out. Utility is only invariant to positive similarity transformations. Hence, utility is measurable on a ratio-scale with full comparability. See d'Aspremont and Gevers (2002) for details.
} 
the following dynamic system:

$$
\begin{aligned}
\dot{c} & =-\frac{u^{\prime}(c)}{u^{\prime \prime}(c)}\left[f^{\prime}(k)-\delta-\rho(k)-\frac{\rho^{\prime}(k)}{\rho(k)}\left(\frac{u(c)}{u^{\prime}(c)}+\dot{k}\right)\right], \\
\dot{k} & =f(k)-c-\delta k .
\end{aligned}
$$

This time, the term in round brackets of equation (23) has the required sign throughout, and it will affect the steady state positively in the case of decreasing discount rates and negatively if one assumes increasing discount rates. Of course, the steady state ought to be affected by this Volterra ratio term due to the recursive nature of the problem. This is in contrast to the model forwarded by Obstfeld (1990) and e.g. Das (2003), where the discount rate is endogenized via consumption and this term is nonexistent. In these standard endogenizations, the optimal steady state can never be to the right of the Golden Rule level. This, of course, is questionable and seems somewhat restrictive, especially when one has the cumulative effect of the discount rate on the utility functional in mind, which can be much more important than the effect of consumption itself. This suggests that if the discount rate is very responsive to changes in wealth, then there ought to be incentive to overaccumulate wealth in comparison to the exogenous discounting case. Overall, in the endogenization via wealth, the policy maker will need to consider how to trade wealth for consumption given this additional feedback of wealth on the discount rate, where higher consumption implies lower wealth and therefore higher discounting.

We now want to analyze the steady state and dynamics of this model. For this we take the steady state versions of (23) and (24) to get

$$
\begin{aligned}
f^{\prime}(k)-\delta-\rho(k) & =\frac{\rho^{\prime}(k)}{\rho(k)} \frac{u(c)}{u^{\prime}(c)}, \\
f(k)-\delta k & =c .
\end{aligned}
$$

We then constrain the domain of wealth to the relevant one only. Firstly, we don't need to concern ourselves with $k>\bar{k}$, where $\bar{k}$ is the level of capital that solves $f(k)=\delta k$. Also, from (25), we know that the steady state curve for consumption is not defined for $k<\underline{k}$, where $\underline{k}>0$ solves $f^{\prime}(k)-\delta=\rho(k)$. Therefore, for the subsequent analysis, it is sufficient to concentrate on the interval $k \in[\underline{k}, \bar{k}]$ only. For simplicity later, we also define $\tilde{k}$, which solves $f^{\prime}(k)=\delta$.

As can be seen from equation (25) the steady state can be either to the left or to the right of the Golden Rule, depending how strongly the discount rate responds to changes in wealth.

The steady state curves have the following shape. Equation (26) is standard and goes from $\{c, k\}=$ $\{0,0\}$ to $\{c, k\}=,\{0, \bar{k}\}$. Equation (25) is only satisfied if, when $k \rightarrow 0 \Longrightarrow c \rightarrow-\infty$; and if when $k \rightarrow \bar{k} \Longrightarrow c>0$. 
Therefore, in the case of a decreasing discount rate, the steady state curve for consumption goes from $-\infty$ to a positive number in the interval $k \in(0, \bar{k}]$. Due to the continuity of both steady state curves for consumption and capital, this implies that a steady state always exists. ${ }^{10}$

We take the total derivative of the steady state curve of capital, equation (25), and consumption, equation (26), to get

$$
\begin{aligned}
\frac{d c}{d k} & =\frac{f^{\prime \prime}(k)-\rho^{\prime}(k)-m(k) \frac{u(c)}{u^{\prime}(c)}}{n(k) \frac{\rho^{\prime}(k)}{\rho(k)}} \\
\frac{d c}{d k} & =f^{\prime}(k)-\delta
\end{aligned}
$$

where $m(k)=\frac{\rho^{\prime \prime}(k) \rho(k)-\rho^{\prime}(k)^{2}}{\rho(k)^{2}}$ with $m(k) \gtrless 0$ and $n(k)=\frac{u^{\prime}(c)^{2}-u^{\prime \prime}(c) u(c)}{u^{\prime}(c)^{2}}>0$. Then the steady state curve (28) has the familiar shape of first increasing, reaching the maximum at $\tilde{k}$, and decreasing thereafter, until crossing the $k$-axis at $\bar{k}$. The denominator of equation (27), given our assumption $u(c) \geq 0$, is negative. The nominator is positive if $f^{\prime \prime}(k)-\rho^{\prime}(k)>m(k) u(c) / u^{\prime}(c)$. We will make use of the subsequent proposition in order to establish in which range of the variables we can sign the nominator.

The following proposition establishes a sufficient condition for a unique steady state. In the rest of the article we are going to concentrate on a unique equilibrium only. The case of multiple steady states has been analyzed by Schumacher (2006).

Proposition 2 A sufficient condition for a unique steady state is given by

$$
f^{\prime \prime}(k)<\rho^{\prime}(k)+m(k) \frac{u(c)}{u^{\prime}(c)}+\left[f^{\prime}(k)-\delta\right] \frac{\rho^{\prime}(k)}{\rho(k)} n(k),
$$

$\forall k \in[\underline{k}, \bar{k}]$. Otherwise, multiple steady states could exist.

\section{Proof 2 See Appendix.}

This sufficient condition for a unique steady state is rather strong and it can be weakened at the cost of tractability. Also, for the case of the exogenous discount rate, this condition reduces to $f^{\prime \prime}(k)<0$, which is the standard concavity condition for the Ramsey model.

Hence, having the preceding sufficiency condition for a unique steady state in mind, equation (29), this implies that given $\rho^{\prime}(k)<0$, then $d c / d k>0$ for $k<\tilde{k}$, and $d c / d k \gtrless 0$ for $k>\tilde{k}$, although if $d c / d k<0$, then the decrease must be slow enough to satisfy the conditions for a unique steady state.

\footnotetext{
${ }^{10}$ This argument however requires the minor assumption of $\rho^{\prime}(k)<\infty, \forall k$.
} 
We can show that the steady state curve for consumption reduces to the one in the Ramsey model. From equation (27) we obtain $d k / d c \rightarrow 0$ when $\rho^{\prime}(k) \rightarrow 0, \forall k$, which is equivalent to the familiar vertial $\dot{c}=0$ line in the Ramsey model. Finally, the steady state curves in case of a unique steady state will approximately take the form as in Figure 1.

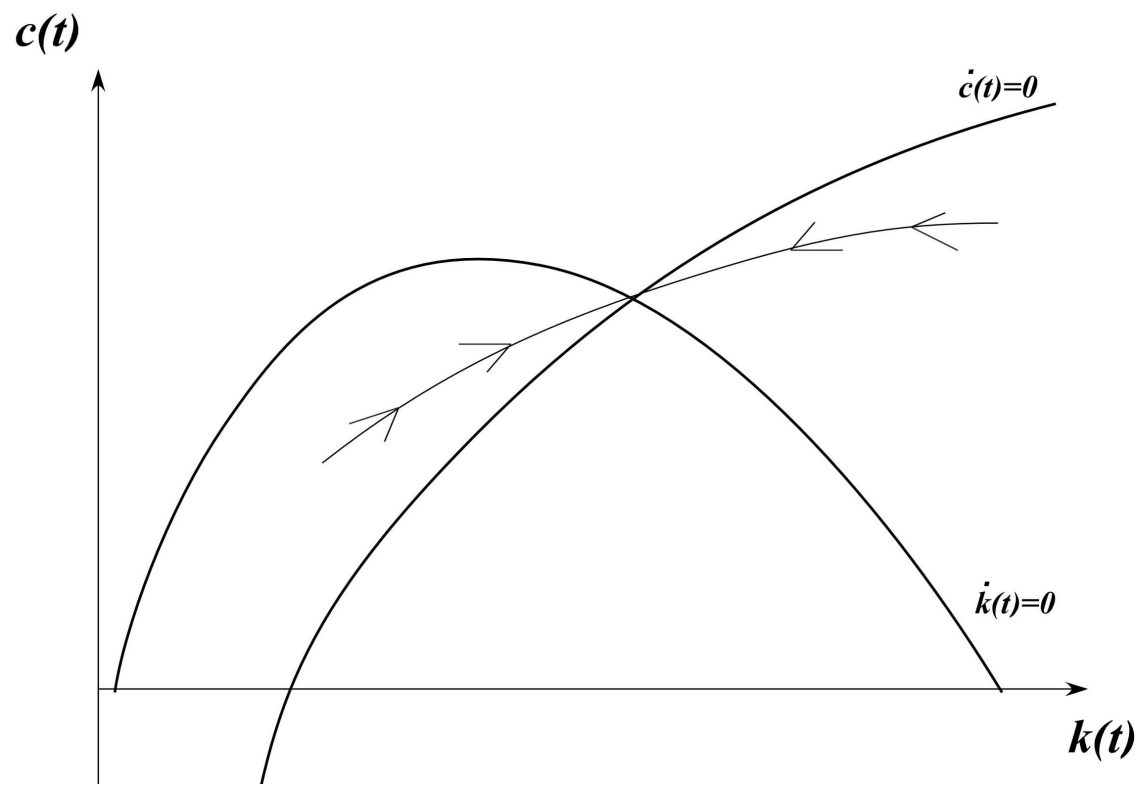

Figure 1: Phase Diagram

We then linearize the system around the unique steady state. This gives the Jacobian of the linearized system

$$
J=\left[\begin{array}{cc}
\rho(k)+\delta-f^{\prime}(k) & -\frac{u^{\prime}(c)}{u^{\prime \prime}(c)}\left(f^{\prime \prime}(k)-\rho^{\prime}(k)-m(k) \frac{u(c)}{u^{\prime}(c)}-\left(f^{\prime}(k)-\delta\right) \frac{\rho^{\prime}(k)}{\rho(k)}\right) \\
-1 & f^{\prime}(k)-\delta
\end{array}\right] .
$$

As is well-known, the system is saddle path stable if there exists one positive and one negative eigenvalue, denoted by $\lambda_{1,2}$. As the trace is $\operatorname{Tr}(J)=\lambda_{1}+\lambda_{2}$, and the determinant is $\operatorname{Det}(J)=\lambda_{1} \lambda_{2}$, it suffices to show that the trace is positive and the determinant is negative. We can thus show that the trace of this matrix is given by $\operatorname{Tr}(J)=\rho(k)>0$, while the determinant is negative if

$$
f^{\prime \prime}(k)<\rho^{\prime}(k)+m(k) \frac{u(c)}{u^{\prime}(c)}+\frac{\rho^{\prime}(k)}{\rho(k)}\left(f^{\prime}(k)-\delta\right) n(k) .
$$

It thus follows trivially that if the sufficient condition for a unique steady state is satisfied then the system is saddle-path stable. 
When comparing the condition for saddle-path stability to the traditional Ramsey case, then for $\rho(k)=\rho$ the above condition reduces to $f^{\prime \prime}(k)<0$, which is again the familiar Ramsey condition. Therefore, this model provides a tractable and direct extension of the Ramsey case.

\section{Can we assure Optimality?}

In the first sections we noticed that the second-order sufficiency conditions, the Mangasarian conditions, require (minimally) a negative utility function. However, as the utility function must be non-negative for intuitively correct results, it is important to check if the Mangasarian conditions can be replaced by a different set of conditions, which nevertheless allow for the existence of an optimal program. This section then deals with two specific ways to derive the existence of an optimal program without resorting to Mangasarian lemma. We then show with a numerical example, utilizing positive utility, that indeed the Mangasarian condition is not necessary for an optimum.

\subsection{Optimality via saddle-path stability}

A simple way to argue that the path obtained by the Pontryagin condition and the transversality condition is globally optimal is by making use of the saddle path stability coupled with the transversality condition. We know by the saddle-path stability of the dynamic system that there exists only one path which satisfies the Pontryagin conditions and the transversality condition. This is the saddle path which leads to the steady state. Any other path will diverge for different initial choices of consumption. This is enough information for optimality. Hence, in this case, the Pontryagin conditions coupled with the transversality condition give rise to an optimal path which maximizes the utility functional, such that $\mathcal{H}\left(k^{*}, c^{*}\right) \geq$ $\mathcal{H}(k, c), \forall\{c, k\}$ which are feasible.

\subsection{Optimality via Bellman's principle of optimality}

It is possible to discretize the model in order to make use of Bellman's equation. Discretizing a continuous time model with recursive preferences has already been used in Drugeon (1996) and Chang (1994).

We can define the value function as

$$
\begin{aligned}
V(k)= & \max _{\{c\}} U(c, k) \quad \text { s.t. } \\
& \dot{k}=f(k)-c-\delta k, \text { where } k(0)>0 \text { given. }
\end{aligned}
$$


The method of discretizing goes as follows. We approximate by splitting the utility integral at time $\epsilon>0$, such that

$$
V(k) \approx \max _{\{c\}_{0}^{\epsilon}}\left\{\int_{0}^{\epsilon} u(c(t)) e^{-\int_{0}^{t} \rho(k(s)) d s} d t+e^{-\int_{0}^{\epsilon} \rho(k(s)) d s} V(k(t+\epsilon))\right\} .
$$

Now, moving $V(k)$ to the right, diving by $\epsilon$ and letting $\epsilon \rightarrow 0$, gives the following discretized version of the above problem:

$$
0=\max _{c}\left\{u(c)-\rho(k) V(k)+V^{\prime}(k) \dot{k}\right\} .
$$

Note that this is not an approximation any longer. In order to arrive at equation (32) we made use of the Leibnitz rule as well as the differentiability of the value function. The differentiability of the value function is not intuitive however, but can be approached indirectly via use of the Volterra derivative (Ryder and Heal 1973). By the convexity of the technology set (see e.g. Becker and Boyd 1992), we know that an optimal path exists.

The first order conditions then give

$$
u^{\prime}(c)=V^{\prime}(k),
$$

and the second-order condition for optimality is

$$
u^{\prime \prime}(c)<0 \text {. }
$$

Due to the concavity of the utility function this implies that second-order conditions go through.

Let us then check whether the optimality conditions are equivalent to the Pontryagin conditions. From equation (33) we know that optimal $c$ is a function of $k$, such that we can write $c(k)$. Substituting this in (32) and differentiating with respect to $k$, plus making use of the envelop theorem, gives

$$
0=-\rho^{\prime}(k) V(k)+V^{\prime \prime}(k) \dot{k}+V^{\prime}(k)\left(f^{\prime}(k)-\delta-\rho(k)\right) .
$$

As is well-known, $V^{\prime}(k)=\lambda$, where $\lambda$ is the shadow price of capital. Also, by application of the chain rule, $V^{\prime \prime}(k) \dot{k}=\frac{\partial V^{\prime}(k)}{\partial k} \frac{\partial k}{\partial t}$, wherefore $V^{\prime \prime}(k) \dot{k}=\dot{\lambda}$. Left to determine is $V(k)$. We can conclude that $V(k)$ is the prospective optimal value of utility. Hence, equation (35) corresponds exactly to equation (23) and therefore the necessary condition derived from Bellman's equation indeed correspond to those from Pontryagin. 


\subsection{A Simulation}

The previous arguments were established to show that the Mangasarian conditions are indeed only sufficient and not necessary to characterize an optimum. We now give a numerical example which provides the last touch to the previous results.

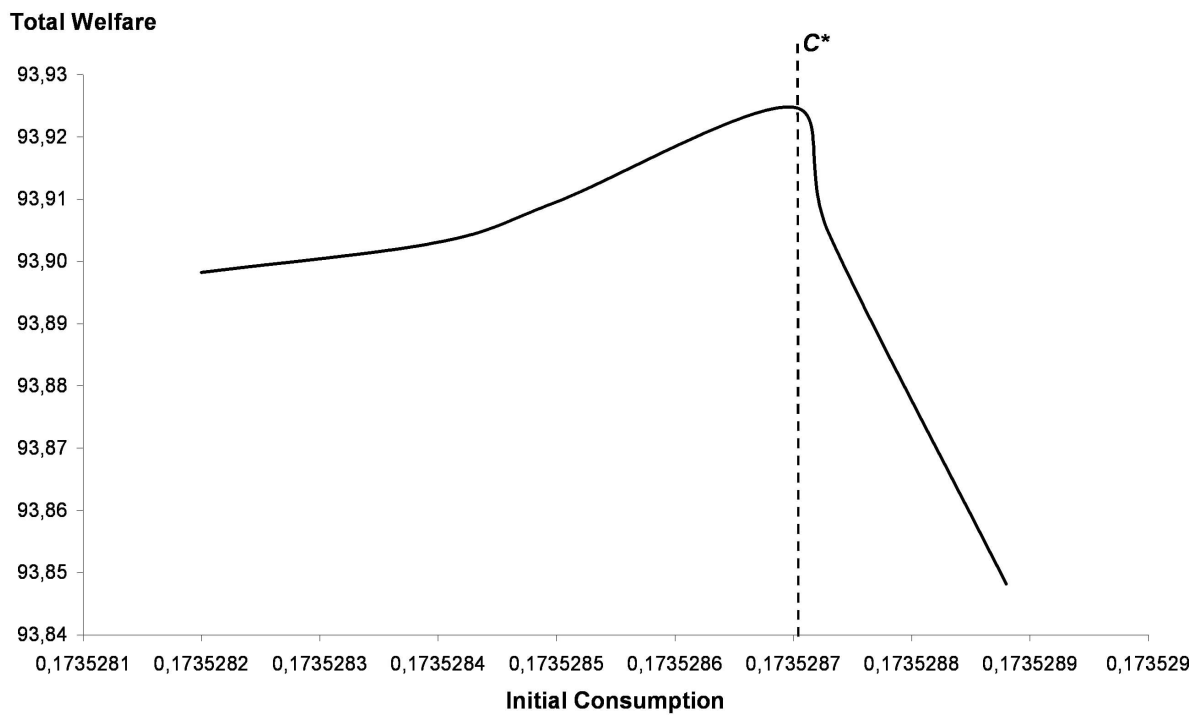

Figure 2: Total Welfare from different initial consumption

We freely choose the following explicit functional forms and parameters: $f(k)=A k^{\alpha}, u(c)=$ $c^{1-\sigma} /(1-\sigma), \rho(k)=\bar{\rho} /(1+\beta k) ; A=0.3, \alpha=0.3, \beta=0.04, \delta=0.05, \bar{\rho}=0.1, \sigma=0.9$. Choosing $\sigma<1$ insures a positive utility throughout. The initial condition for capital is chosen at $k(0)=0.774931$. The unique steady state of this numerical example is given at $\left\{c^{*}, k^{*}\right\}=\{0.268353,2.83411\}$.

Then, Figure 2 shows the total welfare obtainable from different initial values for consumption. The optimal value of consumption, here denoted as $c^{*}$, is derived by the backward integration method introduced in Brunner and Strulik (2000). As can be seen, total welfare increases when converging to the optimal initial value of consumption. Therefore, the simulation supports the prior analysis and indeed suggests that negative utility is not necessary for optimality.

Hence, the fortunate moral of the article is that even though we disregard Mangasarian conditions, the model is now in line with intuition and it can be shown that the dynamic path chosen still leads to an optimum. 


\section{Discussion and Analysis}

This section will utilize the results of the model to assess several specific questions. Firstly, what is the effect of changing technology? Secondly, what can be said about the savings behaviour in this recursive model? Thirdly, what implications has this model on the convergence speed? First things first.

\subsection{The effect of technology}

From the steady state equations we can also draw some conclusions on the effect of a higher level of total factor productivity, A, where we write $f(k)=A g(k)$, with $A>0$. Mathematically, we take partial derivatives of equations (25) and (26) with respect to $k$ and $A$ to get

$$
\begin{aligned}
\frac{d c}{d A} & =\frac{\rho(k) g^{\prime}(k) u^{\prime}(c)^{2}}{\rho^{\prime}(k)\left[u^{\prime}(c)^{2}-u^{\prime \prime}(c) u(c)\right]}<0, \\
\frac{d c}{d A} & =g(k)>0 .
\end{aligned}
$$

Thus, for increases in total factor productivity the $\dot{c}=0$ shifts down whereas the $\dot{k}=0$ line expands. The intuition is that if capital is more productive, then it is more efficient to increase the level of capital and thus to reduce the discount rate (for $\rho^{\prime}(k)<0$ ). An exogenous increase of the marginal effect of capital on the discount rate also shifts the steady state consumption curve down and right, because it is then marginally more efficient to increase capital in order to reduce the discount rate than to increase consumption.

\subsection{Effect on savings behaviour}

The saving rate is given by $s=1-c / f(k)$. We can then calculate the steady state savings rate to assess what influence the endogenous discounting has on savings. We shall do this for the familiar CobbDouglas production function, in which case $f(k) / k=f^{\prime}(k) / \alpha$ holds, as well as for the CRRA utility function. Let us denote steady state savings by $s^{*}$, then it is possible to calculate the steady state savings rate as

$$
s_{e n}^{*}=\frac{\alpha \delta}{\delta+\rho(k)+\frac{\rho^{\prime}(k)}{\rho(k)} \frac{f(k)-\delta k}{1-\sigma}} .
$$

We compare this to the steady state savings rate in the exogenous discounting case, which is $s_{e x}^{*}=\frac{\alpha \delta}{\delta+\rho}$. As $\rho^{\prime}(k)<0$ and $\rho(k)<\rho$, it is clear that steady state savings in the endogenous discounting case is higher than in the exogenous one. 


\subsection{Changes in Convergence speed}

As is well known, the convergence speed in the original Ramsey model is much too high to match the data. Hence, we would like to know whether this model is able to generate a convergence speed more in line with the actual data.

We can approximate the convergence speed with the negative eigenvalue of the linearized system. We know that the trace and determinant are given by $\operatorname{Tr}(J)=\rho(k)>0$ and $\operatorname{Det}(J)=f^{\prime \prime}(k)-\rho^{\prime}(k)-$ $m(k) \frac{u(c)}{u^{\prime}(c)}-\frac{\rho^{\prime}(k)}{\rho(k)}\left(f^{\prime}(k)-\delta\right) n(k)$. We then denote the negative eigenvalue by $\lambda^{e n}<0$, which is given by the formula

$$
\lambda^{e n}=\frac{\rho(k)}{2}-\sqrt{\frac{\rho(k)^{2}}{4}+\frac{u^{\prime}(c)}{u^{\prime \prime}(c)}\left(f^{\prime \prime}(k)-\rho^{\prime}(k)-m(k) \frac{u(c)}{u^{\prime}(c)}-\frac{\rho^{\prime}(k)}{\rho(k)}\left[f^{\prime}(k)-\delta\right] n(k)\right)} .
$$

In comparison, the convergence speed in the Ramsey case, which we denote by $\lambda^{e x}$ for exogenous discounting, is approximately

$$
\lambda^{e x}=\frac{\rho}{2}-\sqrt{\frac{\rho^{2}}{4}+\frac{u^{\prime}(c) f^{\prime \prime}(k)}{u^{\prime \prime}(c)}} .
$$

We shall use specific functional forms as well as common parameter ranges in order to draw some conclusions. The functional forms are $f(k)=A k^{\alpha}, \rho(k)=\frac{\rho}{1+\beta k}, u(c)=\frac{c^{1-\sigma}}{1-\sigma}$, with parameters given by $A=2, \alpha=0.3, \beta=0.03, \delta=0.05, \rho=0.3, \sigma=0.9$. The conditions on the discount rate imply that for extremely poor economies who have no wealth, the discount rate is $\rho=0.3$, whereas for economies at their steady state level the discount rate is approximately $\rho=0.05$. This approximately conforms to empirically verified observations (Frederick et al. 2002).

Mankiw, Phelps and Romer (1995) estimate the convergence speed for the United States. Their rough estimate is at around 4 percent. In comparison to this the convergence speed with the exogenous discounting Ramsey model is approximately 42 percent, which is much too high. The endogenous discounting model presented here gives an approximate convergence speed of 3 percent, which is much more in line with the empirical data.

\subsection{Time consistency}

Models which utilize a variable discount rate are usually subject to problems of time consistency. This, here, is not the case due to the clear recursive nature of the model, wherefore the planner's future self will take the same choices as his current self. Irrespectively of when the planner decides, he will always follow the same course. This, in other words, is time consistency. 
It is possible to imagine a case where the planner is not fully aware of his preference change, such that $\rho(\gamma k)$, where $\gamma \in[0,1]$. If $\gamma=0$, then we have the situation of a completely naïve planner who believes his preferences are not changing. However, when he then "arrives" in the future he notices that his preferences are different from those with which he initially took his decisions and are different from those which he expected to have. In a discrete version of the model with a completely naïve planner (such that $\gamma=0$ ), this implies that the planner takes the decision such that

$$
u\left(c_{1}\right)+\beta u\left(c_{2}\right)+\beta^{2} u\left(c_{3}\right)+\ldots
$$

However, upon arriving at time $t=2$, he notices that his preferences have changed and are actually $\beta(k)>\beta$. Hence the implication is that the preferences from period $t=2$ onwards viewed at $t=1$ are $u\left(c_{2}\right)+\beta u\left(c_{3}\right)$, whereas from period $t=2$ viewed the preferences are $u\left(c_{2}\right)+\beta(k) u\left(c_{3}\right)$. Therefore, equality of marginal (discounted) utility over time does not hold any longer, which implies time inconsistent behaviour and inefficient allocations, as the planner will revise his optimal plans from period to period.

This naïve behaviour is particularly troublesome when decisions span over a very large horizon. For example, if the policy maker believes that his true discount factor is $\beta$, but his actual one $\beta^{\prime}$, and if $\beta=0.9$ and $\beta^{\prime}=0.91$, then these small differences over a horizon of 60 years accumulate to a difference of approximately $100 \%$ in value. Clearly, the closer is $\gamma$ to one, i.e. the less naïve the planner, the more efficient will be his long-run planning.

\section{Conclusion}

In this article we introduce a broad measure of wealth as a source of endogenous discounting. We assess the implications of this endogenization by assuming that wealth decreases the discount rate. The intuition for a decreasing discount rate is that higher wealth is a proxy for the observation that in general, countries with a higher wealth (physical and human) have a lower discount rate. This is due to the fact that physical wealth and human wealth have strong implications on mortality rates, expectations about the future and self-awareness.

We assess the Mangasarian second-order conditions for an optimum and notice that they require negative utility. The same condition is imposed by the Arrow and Kurz conditions. After solving the model by deriving the Pontryagin necessary conditions and obtaining a reduced-form dynamical system, we notice that even though the assumption of a decreasing discount rate is favourable towards the golden 
rule consumption level, we find that, given we impose negative utility as required by the Mangasarian conditions, the steady state is generally further away from the golden rule. We notice that this counterintuitive result is solely due to the negative utility function, an assumption which we find again and again in the models of endogenous discounting. A possible way around this is to dispense looking at Mangasarian sufficiency conditions and simply assume a positive utility function. This way of dealing with the problem has however one drawback. We don't know whether our necessary conditions for optimality are also sufficient for a maximum. This problem we attack in two ways. One, by an argument suggesting that saddle path stability and transversality condition imply there exists only one path which is optimal. Two, by resorting to Bellman's equation, which provides a different sufficiency condition but the same Pontryagin conditions. Finally, we make use of a numerical example which supports our findings that negative utility is not necessary for optimality.

Then we show that even though we disregard the Mangasarian conditions by assuming a positive utility function throughout, we arrive at conclusions which are in line with our intuition. We assess the implications of the model on changes in technology and the implication of endogenous discounting on savings and convergence speed.

Improvements in technology imply a higher level of steady state capital but either a higher or lower level of consumption, depending on whether the steady state was to the left or to the right of the Golden Rule. The direct effect of higher productivity increases the steady state consumption level, whereas the indirect effect via the effect of wealth on discounting reduces the level of steady state consumption.

We notice that the saving rate is higher in the endogenous discounting case and the convergence speed is much slower and in line with empirical evidence, in contrast to the standard Ramsey model and the endogenous discounting case via consumption.

Finally, we discuss the implication of a naïve planner who believes his preferences do not change or do not change completely. We conclude that this behaviour is particularly troublesome and leads to time inconsistent and inefficient allocations.

Overall, this model seems to conform better with empirical evidence than the standard Ramsey model and the method of endogenizing via consumption. 


\section{Appendix}

We use the steady state equations

$$
\begin{aligned}
f^{\prime}(k)-\delta-\rho(k) & =\frac{\rho^{\prime}(k)}{\rho(k)} \frac{u(c)}{u^{\prime}(c)}, \\
f(k)-\delta k & =c .
\end{aligned}
$$

We define $G(k)=A(k)-B(k)$, where $A(k)=f^{\prime}(k)-\delta-\rho(k)$ and $B(k)=\frac{\rho^{\prime}(k)}{\rho(k)} \frac{u(f(k)-\delta k)}{u^{\prime}(f(k)-\delta k)}$. Then $G^{\prime}(k)=f^{\prime \prime}(k)-\rho^{\prime}(k)-m(k) \frac{u(c)}{u^{\prime}(c)}-\left[f^{\prime}(k)-\delta\right] \frac{\rho^{\prime}(k)}{\rho(k)} n(k)$, where $c=f(k)-\delta k$. We know that $\lim _{k \rightarrow 0} G(k)=\infty$ and $\lim _{k \rightarrow \bar{k}} G(k)=z$, where $z$ is a negative but finite number. Hence, the curve $G(k)$ starts from positive infinity to negative finite for $k=\bar{k}$. As each argument of $G(k)$ is continuous, we know that $G(k)$ is continuous. Therefore, a sufficient condition for a unique steady state is then that $G(k)=0$ only for one $k$. This is satisfied when $G^{\prime}(k)<0, \forall k$. This condition holds for $f^{\prime \prime}(k)<\rho^{\prime}(k)+m(k) \frac{u(c)}{u^{\prime}(c)}+\left[f^{\prime}(k)-\delta\right] \frac{\rho^{\prime}(k)}{\rho(k)} n(k)$. 


\section{Bibliography}

Amir, R., "A Characterization of Globally Optimal Paths in the Non-Classical Growth Model," Cowles Foundation Discussion Papers 754, Cowles Foundation, Yale University, 1985.

Ayong Le Kama, A. and K. Schubert, "A note on the consequences of an endogenous discounting depending on the environmental quality," forthcoming in Macroeconomic Dynamics (2005).

Banerjee, A. and A. Newman, "Risk-Bearing and the Theory of Income Distribution," The Review of Economic Studies 58 (1991), 211-235.

Barro, R. and L. Lee, "International Comparisons of Educational Attainment," Journal of Monetary Economics 32 (1993), 363-394.

Barro, R. and X. Sala-i-Martin, Economic Growth, (McGraw-Hill, Inc., 1995).

Becker, R. and J. Boyd III, "Recursive utility and optimal capital accumulation II: sensitivity and duality theory," Economic Theory 2 (1992), 547-563.

Becker, G. and C. Mulligan, “The endogenous determination of time preference," Quarterly Journal of Economics 112 (1997), 729-758.

Böhm-Bawerk, E., Capital and Interest, (South Holland, Libertarian Press, 1889).

Borghans, L. and B. Golsteyn, "Imagination, Time Discounting and Human Capital Investments," mimeo, 2005.

Brunner, M. and H. Strulik, "Solution of perfect foresight saddlepoint problems: A simple method and applications," Journal of Economic Dynamics and Control 26 (2002), 737-753.

Carroll, C., "Buffer-Stock Saving and the Life Cycle/Permanent Income Hypothesis," The Quarterly Journal of Economics 112 (1997), 1-55.

Case, A., D. Lubotsky and C. Paxson, "Economic Status and Health in Childhood: The Origins of the Gradient," The American Economic Review 92 (2002), 1308-1334.

Chang, F., "Optimal Growth and Recursive utility: A Phase-Diagram analysis," Journal of Optimization Theory and Applications 80 (1994), 425-439. 
Das, M., "Optimal growth with decreasing marginal impatience," Journal of Economic Dynamics and Control 27 (2003), 1881-1898.

d'Aspremont, C. and L. Gevers, "Social welfare functionals and interpersonal comparability," in K. Arrow, A. Sen and K. Suzumura, eds., Handbook of Social Choice and Welfare Vol. 1, (Elsevier, 2002), 459-541.

de Walque, D., "Education, information, and smoking decisions : evidence from smoking histories, 1940-2000,” Policy Research Working Paper Series 3362, The World Bank, 2004.

Deaton, A. and C. Paxson, "Intertemporal Choice and Consumption Inequality," Journal of Political Economy 120 (1994), 437-467.

Dechert, D. and K. Nishimura, "A complete characterization of optimal growth paths in an aggregated model with a non-concave production function," Journal of Economic Theory 31 (1983), 332-354.

Drugeon, J.-P., "Impatience and long-run growth," Journal of Economic Dynamics and Control 20 (1996), 281-313.

Elias, J., “The Education Premium Puzzle,” University of Chicago, mimeo, 2003.

Epstein, L. and J. Hynes, "The rate of time preference and dynamic economic analysis," Journal of Political Economy 91 (1983), 611-635.

Epstein, L., “A simple Dynamic General Equilibrium Model," Journal of Economic Theory 41 (1987), 68-95.

Fall, E. and I. Schumacher, "Education as a source of endogenous discounting in a semi-endogenous growth model," mimeo, 2006.

Feldman, J., D. Makuc, J. Kleinman and J. Cornoni-Huntley, "National trends in educational differences in mortality," American Journal of Epidemiology 129 (1989), 919-1033.

Fielding, D. and S. Torres, "Health, Wealth, Fertility, Education and Inequality," University of Otago Economics Discussion Papers No.0505, 2005.

Fisher, I. The Theory of Interest, (Porcupine Pr, Reprint edition, 1930). 
Frederick, S., G. Loewenstein and T. O’Donoghue, "Time Discounting and Time Preference: A Critical Review," Journal of Economic Literature 40 (2002), 351-401.

Grossman, M., "Education and Nonmarket Outcomes," in E. Hanushek and F. Welch, ed., Handbook of the Economics of Education, (Amsterdam, North-Holland, Elsevier, 2003).

Grossman, M. and R. Kaestner, "Effects of education on health," in J. Behrman and N. Stacey, eds., The Social Benefits of Education, (University of Michigan Press, Ann Arbor, Michigan, 1997), 69-123.

Kenkel, D., "Health Behavior, Health Knowledge, and Schooling," Journal of Political Economy 99 (1991), 287-305.

Kitagwa, E. and P. Hauser, Differential Mortality in the United States: A Study in Socioeconomic Epidemiology, (Cambridge, MA: Harvard University Press, 1973).

Koopmans, T., “Stationary Ordinal Utility and Impatience,” Econometrica 28 (1960), 287-309.

Lawrence, E.C., "Poverty and the rate of time preference: evidence from panel data," Journal of Political Economy 99 (1991), 54-75.

Mankiw, G., D. Romer and D. Weil, “A Contribution to the Empirics of Economic Growth,” Quarterly Journal of Economics 107 (1992), 407-437.

Mankiw, G., E. Phels and P. Romer, "The growth of nations," Brookings Papers on Economic Activity 1 (1995), 275-326.

Marshall, A., Principles of Economics, (Macmillan and Co., London, 1890).

Michel, P., “On the Transversality Conditions in Infinite Horizon Problems," Econometrica 50 (1982), 975-985.

Nayga, R. Jr, "Effect of Schooling on Obesity: Is Health Knowledge a Moderating Factor?," Education Economics 9 (2001), 129-137.

Obstfeld, M., "Intertemporal dependence, impatience and dynamics," Journal of Monetary Economics 26 (1990), 45-75.

Pappas, G., S. Queen, W. Hadden and G. Fisher, "The increasing disparity in mortality between socioeconomic groups in the United States, 1960 and 1986," New England Journal of Medicine 329 (1993), 103-109. 
Parfit, D., Reasons and Persons, (Oxford University Press, 1984).

Pigou, A., The Economics of Welfare, (Macmillan and Co., London, 1920).

Pittel, K., "Sustainability and Endogenous Time Preference," in K. Pittel, ed., Sustainability and Endogenous Growth, (Edward Elgar, Cheltenham, UK, 2002).

Portney, P. and J. Weyant, (eds.), Discounting and intergenerational equity, (Resources for the Future, Washington, 1999).

Preston, S. and I. Elo, "Are educational differentials in adult mortality increasing in the United States?," Journal of Aging and Health 7 (1995), 476-496.

Pritchett, L. and L. Summers, "Wealthier is Healthier," The Journal of Human Resources 31 (1996), 841-868.

Ramsey, F., “A Mathematical Theory of Saving,” Economic Journal XXXVIII (1928), 543-559.

Richard, H. and R. Barry, "U.S. life tables for 1990 by sex, race, and education," Journal of Forensic Economics 11 (1998), 9-26.

Ryder, H. and G. Heal, "Optimal Growth with Intertemporally Dependent Preferences," The Review of Economic Studies 40 (1973), 1-31.

Samwick, A., "Discount rate homogeneity and social security reform," Journal of Development Economics 57 (1998), 117-146.

Sander, W., "Schooling and Quitting Smoking," The Review of Economics and Statistics 77 (1995), 191-199.

Schumacher, I., "Endogenous Discounting via Wealth, Twin-Peaks and the Role of Technology," CORE Discussion Paper No. 2006/104, 2006.

Shi, S. and G. Epstein, "Habits and Time Preference," International Economic Review 34 (1993), 61-84.

Scott, M., A new view of economic growth, (Clarendon Press, Oxford, 1989).

Uzawa, H., "Time Preference, the Consumption Function, and optimum Asset Holdings," in J. Wolfe, ed.,Value, Capital and Growth, (Chicago, Chicago University Press, 1968). 
Département des Sciences Économiques de l'Université catholique de Louvain

Institut de Recherches Économiques et Sociales

Place Montesquieu, 3

1348 Louvain-la-Neuve, Belgique 